JouRnal OF Universal Mathematics

Vol.3 No.2 PP.114-120 (2020)

ISSN-2618-5660

DOI: $10.33773 /$ jum. 822384

\title{
NEAR APPROXIMATIONS IN VECTOR SPACES
}

\author{
H. TASBOZAN
}

\begin{abstract}
Near set theory is a concept that emerged from the comparison of similarities between digital images. Another set theory, soft set, was obtained by Molodstov[3]. The space where we obtain the near sets is the nearness approximation space. Tasbozan [25] introduce the soft sets based on a near approximation space. The group and ring structure of algebraic structures on near sets are defined. In this article, the structure of vector spaces will be discussed with close approximations. First I defined a pair of near approximation operators with respect to congruence based on a subspace, investigated near subsets in the vector space and its basic properties. Then considered a near soft set over a vector space has a properties of vector space defined lower and upper near soft approximation operators in vector spaces.
\end{abstract}

\section{INTRODUCTION}

The rough set was created by pawlak with an equivalence relation on the universal set.[14, 15]. The remaining part of the equivalence classes in this equivalence relation is defined as the upper approximation of the rough set and the part that does not intersect with the lower approximation equivalence class is empty. The rough set is expressed by a pair of these approximations. The concept of near set, which is a more general form of the rough set, was built by pawlak[18, 17, 20, 21, 22, 23, 24, 19]. Accordingly, each rough set is a near set. In near sets, equivalence classes are given directly using real-valued functions instead of tables from which equivalence classes in rough sets are obtained. That's less complicated than that. By trying to make transitions between these theories, different sets have been obtained by studying the theories together. Many studies have been done in the field of algebraic structure as well as the studies in engineering field with these theories.

The concept of soft set, which is another different set theory, was found by Molodstov[3] and studied by many authors $[2,4,5]$. This set theory has been highly recognized in both theoretical and engineering. Feng and li[1] have obtained the rough soft set by associating the concepts of rough set and soft set, and similarly, Tasbozan[25] has obtained the near soft set builts up a connection between soft set and near set. The algebraic side of the rough set has been studied by many

Date: November 6, 2020.

2000 Mathematics Subject Classification. 20 N02.

Key words and phrases. Soft sets, Near sets, Near soft sets, Near subsets in the vector space, Near soft vector space. 
scientists $[7,6,26,27,10,11,13,12,8,9]$. Biswas and Nanda made the definition of subgroup with the approximation and Kuroki[12] made the definition of sub-ring with the approximation. Wang [8] studied some properties of the approximations for normal subgroups. Among the algebraic studies in near sets, firstly, Bağırmaz[11] gave near approximations on groups and davvas[6] gave near approximations on rings which is another one. My goal in this article is to create some properties of vector space on near approximation spaces. The notions of near subsets in the vector space and near soft approximation operators in vector spaces are defined and their basic properties are investigated.

\section{PRELiminary}

2.1. Congruences and vector subspaces. A congruence relation $\delta$ defined on the vector space $V$ and the properties provided for a subspace $W$ of this vector space are known by [26, 27].

Proposition 1. Let $W, W_{1}, W_{2}$ be subspaces of $V$. Then for all $\alpha, \beta \in V$,

(1) $\delta_{W}(\alpha)+\delta_{W}(\beta)=(\alpha+W)+(\beta+W)=\delta_{W}(\alpha+\beta)$

(2) $\delta_{W}(k \alpha)=(k \alpha+W)=k \delta_{W}, 0 \neq k \in K$.

(3) $\delta_{W 1+W_{2}}(\alpha+\beta)=\delta_{W_{1}}(\alpha)+\delta_{W 2}(\beta)$.

(4) $\delta_{W 1 \cap W_{2}}(\alpha)=\delta_{W_{1}}(\alpha) \cap \delta_{W 2}(\alpha) \cdot[27]$

\subsection{Near Sets.}

Definition 2.1. Let $\left(\mathcal{O}, F, \sim_{B_{r}}, N_{r}, v_{N_{r}}\right)=N A S$ be an nearness approximation space where $\mathcal{O}$ is a nonempty set of perceptual objects, $F$ is a countable set of probe functions and $B \subseteq F$. The sets

$$
\begin{aligned}
& \text { (1): } N_{r}(B)_{*} X=\bigcup_{x:[x]_{B_{r}} \subseteq X}[x]_{B_{r}}=\left\{x \in \mathcal{O}:[x]_{B_{r}} \subseteq X\right\}, \\
& \text { (2): } N_{r}(B)^{*} X=\bigcup_{x:[x]_{B_{r}} \cap X \neq \emptyset}[x]_{B_{r} .}=\left\{x \in \mathcal{O}:[x]_{B_{r}} \cap X \neq \emptyset\right\}
\end{aligned}
$$

(3): $\operatorname{Bnd}_{N_{r}(B)}(X)=N_{r}(B)^{*} X \backslash N_{r}(B)_{*} X=\left\{x \in N_{r}(B)^{*} X: x \notin N_{r}(B)_{*} X\right\}$, are called $N_{r}(B)$-lowerapproximation $N_{r}(B)_{*} X, N_{r}(B)$-upperapproximation $N_{r}(B)^{*} X$ and boundary region of $X$ in $\left(\mathcal{O}, F, \sim_{B_{r}}, N_{r}, v_{N_{r}}\right)$, respectively. A set $X$ with an approximation boundary $\left|B n d_{N_{r}(B)}(X)\right| \geq 0$ is a near set[25].

\subsection{Soft Sets.}

Definition 2.2. Let $O$ be an objects set and $F$ be a properties of objects in $O$ and $\mathcal{P}(O)$ is the set of all subsets of $O$. A pair $(K, B)$ is called soft set over $O$ if $B \subseteq F$ and $K: B \rightarrow \mathcal{P}(O)[4]$.

\subsection{Near Soft Sets.}

Definition 2.3. Let $N A S$ be a nearness approximation space and $\sigma=(F, B)$ be a soft set over $\mathcal{O}$ where $\mathcal{O}$ is a nonempty set of perceptual objects, $F$ is a countable set of probe functions and $B \subseteq F$. The sets $N_{r}^{*}(\sigma)=\left(F^{*}, B\right), N_{r} *(\sigma)=\left(F_{*}, B\right)$ are called the upper and lower near approximation of $\sigma=(F, B)$ in $N A S$.

(1): $F_{*}(\phi)=N_{r} *(F(\phi))=\cup\left\{x \in \mathcal{O}:[x]_{B r} \subseteq F(\phi)\right\}$, 
(2): $F^{*}(\phi)=N_{r}^{*}(F(\phi))=\cup\left\{x \in \mathcal{O}:[x]_{B r} \cap F(\phi) \neq \emptyset\right\}$ where all $\phi \in B$.

: Then the soft set $\sigma=(F, B)$ is called a near soft set and denotes $N S S$ [25].

2.5. Near subsets of vector spaces. Let $V$ be a vector space over $K, Z$ be a subspace of $V$, and $A$ a nonempty subset of $V . \delta$ is a congruence on $V, \delta=\delta_{Z}$ where $Z=[0]_{\delta} . \delta_{Z}$ is denote a congruence determined by a subspace $Z$.

The sets $N_{r \delta *}(A)=\{\alpha \in V: \delta(\alpha) \subseteq A\}$ and $N_{r} \delta^{*}(A)=\{\alpha \in V: \delta(\alpha) \cap A \neq \emptyset\}$ are called $\delta$-lower and $\delta$-upper near approximations of a set $A$ with respect to congruence relation $\delta$, respectively. If $B n d N_{r} \delta(A)=N_{r}^{*} \delta(A) \backslash N_{r *} \delta(A) \geq 0, A$ is called a near set in vector space.

Definition 2.4. Let $Z$ be a subspace of vector space $V$, and $A \subseteq V . \delta=\delta_{Z}$ where $Z=[0]_{\delta}$ and $\delta(\alpha)=\delta_{Z}(\alpha)=\alpha+Z$ for any $\alpha \in V$. Then $\delta_{r}=\delta_{Z r}=\alpha+Z_{r}$ $=[\alpha]_{Z_{r}}$ The sets

$$
\begin{aligned}
& N_{r *}(Z)(A)=\left\{\alpha \in V: \alpha+Z=[\alpha]_{Z_{r}} \subseteq A\right\} \\
& N_{r}{ }^{*}(Z)(A)=\left\{\alpha \in V:[\alpha]_{Z_{r}} \cap A \neq \emptyset\right\}
\end{aligned}
$$

are called the lower and upper near approximations of a set $A$ with respect to subspace $Z$ respectively. $(V, Z)$ is called a near approximation space.

Example 2.5. Let $V=Z_{12}$ be a vector space on $K$. Where $K=\{0,1\}$ is two elements field. $Z_{1}=\{0,6\}, Z_{2}=\{0,4,8\}$ is a subspace of $V$. $A=\{0,2,3,6,8,9,10\}$. Then

$$
\begin{aligned}
\text { For } r=1, \quad & \delta_{r}=\delta_{Z r}=\alpha+Z_{r}=[\alpha]_{Z_{r}}, \quad \delta_{1}=\left\{[\alpha]_{Z_{1}},[\alpha]_{Z_{2}}\right\}, \\
\delta_{Z_{1}} & =\alpha+Z_{1}=[\alpha]_{Z_{1}},[0]_{Z_{1}}=\{0,6\},[1]_{Z_{1}}=\{1,7\}, \\
{[2]_{Z_{1}} } & =\{2,8\},[3]_{Z_{1}}=\{3,9\},[4]_{Z_{1}}=\{4,10\},[5]_{Z_{1}}=\{5,11\} \\
\delta_{Z_{2}} & =\alpha+Z_{2}=[\alpha]_{Z_{2}},[0]_{Z_{2}}=\{0,4,8\},[1]_{Z_{2}}=\{1,5,9\}, \\
{[2]_{Z_{2}} } & =\{2,6,10\},[3]_{Z_{2}}=\{3,7,11\}
\end{aligned}
$$

$N_{1 *}(Z)(A)=\left\{\alpha \in V: \alpha+Z=[\alpha]_{Z_{r}} \subseteq A\right\}=\{0,3,6,8,9\}, \quad N_{1}{ }^{*}(Z)(A)=\{\alpha \in$ $\left.V:[\alpha]_{Z_{r}} \cap A \neq \emptyset\right\}=Z_{12}, \quad B n d N_{1}(Z)(A)=Z_{12} \backslash\{0,3,6,9\} \geq 0$.

for $r=2, \quad \delta_{r}=\delta_{Z r}=\alpha+Z_{r}=[\alpha]_{Z_{r}}, \quad \delta_{2}=\left\{[\alpha]_{\left\{Z_{1}, Z_{2}\right\}}\right\},\left\{Z_{1}, Z_{2}\right\}=$ $\{0,4,6,8\}$

$$
\begin{aligned}
\delta_{\left\{Z_{1}, Z_{2}\right\}} & =\alpha+\left(\left\{Z_{1}, Z_{2}\right\}\right)=[\alpha]_{\left\{Z_{1}, Z_{2}\right\}},[0]_{Z_{1}}=\{0,4,6,8\}, \\
{[1]_{\left\{Z_{1}, Z_{2}\right\}} } & =\{1,5,7,9\},[2]_{\left\{Z_{1}, Z_{2}\right\}}=\{2,6,8,10\},[3]_{\left\{Z_{1}, Z_{2}\right\}}=\{3,7,9,11\},
\end{aligned}
$$

$N_{2 *}(Z)(A)=\left\{\alpha \in V: \alpha+Z=[\alpha]_{Z_{r}} \subseteq A\right\}=\{2,6,8,10\}, \quad N_{1}^{*}(Z)(A)=$ $\left\{\alpha \in V:[\alpha]_{Z_{r}} \cap A \neq \emptyset\right\}=Z_{12}, \quad \operatorname{BndN}_{1}(Z)(A)=Z_{12} \backslash\{2,6,8,10\} \geq 0$. Then $A$ becomes a near set in vector space.

Theorem 2.6. Let $Z$ be a subspace of $V, A$ and $B$ nonempty subsets of $V$. Then

(1) $N_{r *}(Z)(A) \subseteq A \subseteq N_{r}^{*}(Z)(A)$

(2) $N_{r *}(Z)(A \cap B)=N_{r *}(Z)(A) \cap N_{r *}(Z)(B)$

(3) $N_{r}^{*}(Z)(A \cup B)=N_{r}^{*}(Z)(A) \cup N_{r}^{*}(Z)(B)$

(4) $N_{r *}(Z)(A \cup B) \supseteq N_{r *}(Z)(A) \cup N_{r *}(Z)(B)$

(5) $N_{r}^{*}(Z)(A \cap B)=N_{r}^{*}(Z)(A) \cap N_{r}^{*}(Z)(B)$

(6) $A \subseteq B$ then $N_{r *}(Z)(A) \subseteq N_{r *}(Z)(B), N_{r}^{*}(Z)(A) \subseteq N_{r}^{*}(Z)(B)$. 
Example 2.7. Let $V=Z_{12}$ be a vector space on $K$. Where $K=\{0,1\}$ is two elements field. $Z_{1}=\{0,6\}, Z_{2}=\{0,4,8\}$ is a subspace of $V . A=\{0,2,3,6,8,9,10\}, . B=$ $\{1,5,7,9\}$ Then

$$
\begin{aligned}
\text { For } r=1, \quad & \delta_{r}=\delta_{Z_{r}}=\alpha+Z_{r}=[\alpha]_{Z_{r}}, \quad \delta_{1}=\left\{[\alpha]_{Z_{1}},[\alpha]_{Z_{2}}\right\}, \\
\delta_{Z_{1}} & =\alpha+Z_{1}=[\alpha]_{Z_{1}},[0]_{Z_{1}}=\{0,6\},[1]_{Z_{1}}=\{1,7\}, \\
{[2]_{Z_{1}} } & =\{2,8\},[3]_{Z_{1}}=\{3,9\},[4]_{Z_{1}}=\{4,10\},[5]_{Z_{1}}=\{5,11\} \\
\delta_{Z_{2}} & =\alpha+Z_{2}=[\alpha]_{Z_{2}},[0]_{Z_{2}}=\{0,4,8\},[1]_{Z_{2}}=\{1,5,9\}, \\
{[2]_{Z_{2}} } & =\{2,6,10\},[3]_{Z_{2}}=\{3,7,11\}
\end{aligned}
$$

$N_{1 *}(Z)(A)=\left\{\alpha \in V: \alpha+Z=[\alpha]_{Z_{r}} \subseteq A\right\}=\{0,3,6,8,9\}, \quad N_{1 *}(Z)(B)=\{1,7\}$ $N_{1}{ }^{*}(Z)(A)=\left\{\alpha \in V:[\alpha]_{Z_{r}} \cap B \neq \emptyset\right\}=Z_{12}, N_{1}{ }^{*}(Z)(B)=\{1,3,5,7,9,11\}$ $\operatorname{BndN}_{1}(Z)(A)=Z_{12} \backslash\{0,3,6,9\} \geq 0 . \operatorname{BndN}_{1}(Z)(B)=\{1,3,5,7,9,11\} \backslash\{1,7\} \geq$ 0 .

for $r=2, \quad \delta_{r}=\delta_{Z r}=\alpha+Z_{r}=[\alpha]_{Z_{r}}, \quad \delta_{2}=\left\{[\alpha]_{\left\{Z_{1}, Z_{2}\right\}}\right\},\left\{Z_{1}, Z_{2}\right\}=$ $\{0,4,6,8\}$

$$
\delta_{\left\{Z_{1}, Z_{2}\right\}}=\alpha+\left(\left\{Z_{1}, Z_{2}\right\}\right)=[\alpha]_{\left\{Z_{1}, Z_{2}\right\}},[0]_{Z_{1}}=\{0,4,6,8\},[1]_{\left\{Z_{1}, Z_{2}\right\}}=\{1,5,7,9\},
$$$$
[2]_{\left\{Z_{1}, Z_{2}\right\}}=\{2,6,8,10\},[3]_{Z_{1}}=\{3,7,9,11\},
$$

$N_{2 *}(Z)(A)=\left\{\alpha \in V: \alpha+Z=[\alpha]_{Z_{r}} \subseteq A\right\}=\{2,6,8,10\}, \quad N_{1}{ }^{*}(Z)(A)=\{\alpha \in$ $\left.V:[\alpha]_{Z_{r}} \cap A \neq \emptyset\right\}=Z_{12}, \quad B n d N_{1}(Z)(A)=Z_{12} \backslash\{2,6,8,10\} \geq 0 . N_{2 *}(Z)(B)=$ $\left\{\alpha \in V: \alpha+Z=[\alpha]_{Z_{r}} \subseteq B\right\}=\{1,5,7,9\}, \quad N_{1}^{*}(Z)(B)=\left\{\alpha \in V:[\alpha]_{Z_{r}} \cap B \neq\right.$ $\emptyset\}=\{1,5,7,9\}, \quad B n d N_{1}(Z)(B)=\emptyset \geq 0$. Then $A, B$ is a $N S S$ in vector space. Then the following statements are true;

(1) $N_{r *}(Z)(A) \subseteq A \subseteq N_{r}^{*}(Z)(A) \quad, \quad\{0,3,6,8,9\} \subseteq\{0,2,3,6,8,9,10\} \subseteq Z_{12}$

(2) $N_{r *}(Z)(A \cap B)=N_{r *}(Z)(A) \cap N_{r *}(Z)(B)=\emptyset$

(3) $N_{r}^{*}(Z)(A \cup B)=N_{r}^{*}(Z)(A) \cup N_{r}^{*}(Z)(B)=Z_{12}$

(4) $N_{r *}(Z)(A \cup B) \supseteq N_{r *}(Z)(A) \cup N_{r *}(Z)(B)=\{0,1,3,6,7,8,9\}$

(5) $N_{r}^{*}(Z)(A \cap B)=N_{r}^{*}(Z)(A) \cap N_{r}^{*}(Z)(B)=\{1,3,5,7,9,11\}$

Theorem 2.8. Let $Z$ be a subspace of $V, A$ and $B$ nonempty subspaces of $V$. Then

(1) $N_{r}^{*}(Z)(A+B)=N_{r}^{*}(Z)(A) \cup N_{r}^{*}(Z)(B)$

(2) $N_{r *}(Z)(A+B) \supseteq N_{r *}(Z)(A)+N_{r *}(Z)(B)$

(3) $N_{r}^{*}(Z)(k A)=k N_{r}^{*}(Z)(A)$ for any $0 \neq k \in K$

(4) $N_{r *}(Z)(k A)=k N_{r *}(Z)(A)$ for any $0 \neq k \in K$.

Theorem 2.9. Let $Z$ and $W$ be a subspace of $V, A$ and $B$ nonempty subspaces of V.Then

(1) $N_{r *}(Z \cap W)(A) \supseteq N_{r *}(Z)(A) \cup N_{r *}(W)(A)$

(2) $N_{r}^{*}(Z \cap W)(A) \subseteq N_{r}^{*}(Z)(A) \cap N_{r}^{*}(W)(A)$

(3) $N_{r}^{*}(Z+W)(A+B)=N_{r}^{*}(Z)(A)+N_{r}^{*}(W)(B)$

(4) $N_{r *}(Z+W)(A+B) \supseteq N_{r *}(Z)(A)+N_{r *}(W)(B)$

(5) $Z \subseteq W \Rightarrow N_{r *}(Z)(A) \subseteq N_{r *}(Z)(A)$ and $N_{r *}(Z)(A) \subseteq N_{r *}(W)(A)$.

2.6. Near soft approximations in vector spaces. Let $\sigma=(F, B)$ be a $N S S, \mathcal{O}$ be a vector space over a field $K$ and $B$ be the parameter set. Let $\sigma_{1}=\left(F_{1}, B\right), \sigma_{2}=$ $\left(F_{2}, B\right), \ldots, \sigma_{n}=\left(F_{n}, B\right)$ be $n N S S$ in $(\mathcal{O}, B)$. Then $(F, B)=\sigma_{1}+\sigma_{2}+\ldots+\sigma_{n}$ is a $N S S$ over $(\mathcal{O}, B)$ and is defined as $F(\phi)=\left\{x_{1}+x_{2}+\ldots+x_{n}: x_{i} \in F_{i}(\phi), i=\right.$ $1,2, \ldots, n\}$ for each $\phi \in B$. Let $\alpha \in K$ be any scalar and $\sigma=(F, B)$ be a $N S S$ over 
$(\mathcal{O}, B)$, then $\alpha F$ is a $N S S$ over $(\mathcal{O}, B)$ and is defined as follows: $\alpha(F, B)=(G, B)$, $G(\phi)=\{\alpha x: x \in F(\phi)\}$ for each $\phi \in B$.

Definition 2.10. Let $\sigma=(F, B)$ be a $N S S$ over $\mathcal{O}$ in $N A S$ and $\mathcal{O}$ be a vector space over a field $K$ and $B$ be the parameter set. If $(F, B)=\sigma_{1}+\sigma_{2}+\ldots+\sigma_{n}$ is a $N S S$ over $(\mathcal{O}, B)$ and is defined as $F(\phi)=\left\{x_{1}+x_{2}+\ldots+x_{n}: x_{i} \in F_{i}(\phi), i=1,2, \ldots, n\right\}$ for each $\phi \in B$ and $\alpha \in K$ be any scalar, $\alpha F$ is a $N S S$ over $(\mathcal{O}, B)$ and is defined as follows: $\alpha(F, B)=(G, B), G(\phi)=\{\alpha x: x \in F(\phi)\}$ for each $\phi \in B$. Then $N_{r}^{*}(\sigma)$, $N_{r} *(\sigma)$ are called the upper and lower near soft approximation operators in vector spaces, respectively and $(F, B)$ called as near soft vector space.

Example 2.11. Let $\mathcal{O}=Z_{12}$ be a vector space over a field $K$ and $B=\left\{\phi_{1}, \phi_{2}\right\} \subseteq$ $\mathcal{F}=\left\{\phi_{1}, \phi_{2}\right\}$ be a set of functions. $\phi_{1}=\{0,4,8\}, \phi_{2}=\{0,6\}$ Let $\sigma_{1}=\left(F_{1}, B\right), \sigma_{2}=$ $\left(F_{2}, B\right)$ be near soft sets in $(\mathcal{O}, B) .(F, B)=\left\{\sigma_{1}, \sigma_{2}\right\}=\left\{\left(F_{1}, B\right),\left(F_{2}, B\right)\right\}$

Let $\sigma_{1}=\left(F_{1}, B\right), B=\left\{\phi_{1}, \phi_{2}\right\}$ be a soft set defined by

$$
F_{1}\left(\phi_{1}\right)=\{0,4\}, F_{1}\left(\phi_{2}\right)=\{0,6,8\}
$$

. for $r=1,\left(F_{1}, B\right)$ is a $N S S$;

$[0]_{\phi_{1}}=\{0,4,8\},[1]_{\phi_{1}}=\{1,5,9\},[2]_{\phi_{1}}=\{2,6,10\},[3]_{\phi_{1}}=\{3,7,11\}$

$[0]_{\phi_{2}}=\{0,6\},[1]_{\phi_{2}}=\{1,7\},[2]_{\phi_{2}}=\{2,8\},[3]_{\phi_{2}}=\{3,9\},[4]_{\phi_{2}}=\{4,10\},[5]_{\phi_{2}}=\{5,11\}$

$$
N_{1 *}\left(\sigma_{1}\right)=N_{1 *}\left(F_{1}(\phi), B\right)=\left(N_{1 *} F_{1}(\phi), B\right)=\left(F_{1 *}(\phi), B\right)
$$

for $\phi_{2} \in B, N_{1 *}(\sigma)=\left(F_{1 *}\left(\phi_{2}\right), B\right)=\left\{\left(\phi_{2},\{0,6\}\right)\right\}$

$$
N_{1}^{*}\left(\sigma_{1}\right)=\left(F_{1}^{*}(\phi), B\right)
$$

for $\phi_{1}, \phi_{2} \in B, N_{1}^{*}(\sigma)=\left\{\left(\phi_{1},\{0,4,8\}\right),\left(\phi_{2},\{0,2,6,8\}\right)\right\}$

$\operatorname{Bnd}_{N}(\sigma) \geq 0$, then $\left(F_{1}, B\right)$ is a $N S S$.

$r=2,\left(F_{1}, B\right)$ is a $N S S$;

$$
\begin{aligned}
{[0]_{\phi_{1}, \phi_{2}}=\{0\}, } \\
{[1]_{\phi_{1}, \phi_{2}}=\{1\}, \ldots } \\
{[11]_{\phi_{1}, \phi_{2}}=\{11\} } \\
N_{2 *}\left(\sigma_{1}\right)=\left\{\left(\phi_{1},\{0,4\}\right),\left(\phi_{2},\{0,6,8\}\right)\right\} \\
N_{2}^{*}\left(\sigma_{1}\right)=\left\{\left(\phi_{1},\{0,4\}\right),\left(\phi_{2},\{0,6,8\}\right)\right\}
\end{aligned}
$$

$\operatorname{Bnd}_{N}(\sigma) \geq 0$, then $\sigma_{1}=\left(F_{1}, B\right)$ is a NSS.

Let $\sigma_{2}=\left(F_{2}, B\right), B=\left\{\phi_{1}, \phi_{2}\right\}$ be a soft set defined by

$$
F_{2}\left(\phi_{1}\right)=\{0,8\}, F_{2}\left(\phi_{2}\right)=\{1,2,7\}
$$

. for $r=1,\left(F_{2}, B\right)$ is a $N S S$;

$$
N_{1 *}\left(\sigma_{2}\right)=N_{1 *}\left(F_{2}(\phi), B\right)=\left(N_{1 *} F_{2}(\phi), B\right)=\left(F_{2 *}(\phi), B\right)
$$

for $\phi_{2} \in B, N_{1 *}\left(\sigma_{2}\right)=\left(F_{2 *}\left(\phi_{2}\right), B\right)=\left\{\left(\phi_{2},\{1,7\}\right)\right\}$

$$
N_{1}^{*}\left(\sigma_{2}\right)=\left(F_{2}^{*}(\phi), B\right)
$$


for $\phi_{1}, \phi_{2} \in B, N_{1}^{*}\left(\sigma_{2}\right)=\left\{\left(\phi_{1},\{0,4,8\}\right),\left(\phi_{2},\{1,2,7,8\}\right)\right\}$

$\operatorname{Bnd}_{N}(\sigma) \geq 0$, therefore $\left(F_{2}, B\right)$ is a $N S S$.

Lets see if $(F, B)$ is a near soft vector space.

$*:(F, B)=\sigma_{1}+\sigma_{2}=\left(F_{1}, B\right)+\left(F_{2}, B\right)=\left\{\left(\phi_{1},\{0,4\}\right),\left(\phi_{2},\{0,6,8\}\right)\right\} \cup$ $\left\{\left(\phi_{1},\{0,8\}\right),\left(\phi_{2},\{1,2,7\}\right)\right\}=\left\{\left(\phi_{1},\{0,4,8\}\right),\left(\phi_{2},\{1,2,6,7,8\}\right)\right\}$ is a $N S S$.

$N_{1 *}(F, B)=\left\{\left(\phi_{1},\{0,4,8\}\right),\left(\phi_{2},\{1,2,6,7,8\}\right)\right\}=N_{1}^{*}(F, B), \operatorname{Bnd}_{N}(F, B) \geq 0$

*: $\alpha=2 \in Z$ be any scalar and $\sigma_{1}=\left(F_{1}, B\right)$ is a $N S S$. Then

$\alpha \sigma_{1}=\alpha\left(F_{1}, B\right)=(G, B)=2 .\left\{\left(\phi_{1},\{0,4\}\right),\left(\phi_{2},\{0,6,8\}\right)\right\}=\left\{\left(\phi_{1},\{0,8\}\right),\left(\phi_{2},\{0,4\}\right)\right\}$

$N_{1 *}(G, B)=\emptyset$ therefore $(G, B)$ is not a $N S S$. Because of this $\sigma=(F, B)$

is not a near soft vector space .

Definition 2.12. Let $\mathcal{O}$ be a vector space over a field $K$ and $B$ be a parameter set. Let $\sigma=(F, B)$ be a $N S S$ over $\mathcal{O}$. Then $G$ is said to be a near soft vector space(denotes $N S V S$ ) or a near soft linear space of $\mathcal{O}$ over $K$, if $G(\phi)$ is a vector subspace of $\mathcal{O}$, for each $\phi \in B$.

Definition 2.13. Let $\sigma=(F, B)$ be a $N S V S$ of $\mathcal{O}$ over $K$ and $\operatorname{Bnd}_{N}(F, B) \geq 0$.

i) $(F, B)$ is said to be null $N S V S$ if $F(\phi)=\{\varphi\}$,for each $\phi \in B$ where $\varphi$ is null element of $\mathcal{O}$.

ii) $(F, B)$ is said to be absolute $N S V S$ if $F(\phi)=\mathcal{O}$, for each $\phi \in B$.

Definition 2.14. Let $\sigma=(F, B)$ be a $N S V S$ of $\mathcal{O}$ over $K$.Let $\sigma=(G, B)$ be a $N S S$ over $(\mathcal{O}, B)$. Then $(G, B)$ is said to be a $N S V S$ of $(F, B)$ if it satistified the following conditions;

i) for each $\phi \in B, G(\phi)$ is a vector subspace of $\mathcal{O}$ over $K$.

ii) $F(\phi) \supseteq G(\phi)$ for each $\phi \in B$.

\section{Conclusions}

In this study, we introduce near subsets in the vector space and near soft approximation operators in vector spaces. Based on the relationships between the congruences and vector subspaces of a vector space, I have given the definition of the lower approximations in the near approximation spaces based on a vector subspace of a subset of the vector space. I think the near soft vector spaces given here will be useful in theory as well as applications of near sets and soft sets. I will try to obtain different results and findings regarding these concepts in my future studies.

\section{REFERENCES}

[1] Feng, F., Li, C., Davvaz, B., Ali, M.I.: Soft sets combined with fuzzy sets and rough sets, Soft Comput., 14, 2010, 899-911.

[2] Simsekler, T., Yuksel, S.: Fuzzy soft topological spaces. Annals of Fuzzy Mathematics and Informatics, 5, 2013, 87-96.

[3] Molodtsov, D.: Soft set theory-first results, Computers and Mathematics with Applications, 37, 1999, 19-31.

[4] Aktas, H., Cagman, N.: Soft sets and soft groups, Information Sciences, 177, 2007, 2726-2735.

[5] Maji, P. K., Biswas, R. and Roy, A.R.: Soft set theory, Computers and Mathematics with Applications, 45, 2003, 555-562.

[6] B. Davvaz, Roughness in rings, Inform. Sci. 164 (2004) 147-163. 
[7] B.Davvaz, Soleha, D.W. Setyawati, Near approximations in rings, AAECC, 30 (2020) Doi: 10.1007/S00200-020-00421-3.

[8] C.Z. Wang, D.G. Chen, A short note on some properties of rough groups, Comput. Math. Appl. 59 (2010)431-436.

[9] D. Miao, S. Han, D. Li, and L. Sun, Rough Group, Rough Subgroup and Their Properties, D. Ślkezak et al. (Eds.): RSFDGrC 2005, LNAI 3641, pp. 104-113, 2005.C) Springer-Verlag Berlin Heidelberg, 2005.

[10] N. Bağırmaz, A. F. Özcan, Rough semigroups on approximation spaces, International Journal of Algebra, Vol. 9, 2015, no. 7, 339-350.

[11] N. Bağırmaz, Near approximations in groups, AAECC, 30 (2019) 285-297.

[12] N. Kuroki, P.P. Wang, The lower and upper approximations in a fuzzy group, Information Sciences 90 (1996) 203-220.

[13] R. Biswas, S. Nanda, Rough groups and rough subgroups, Bull. Polish Acad. Sci. Math 42 (1994) 251-254.

[14] Z. Pawlak, Rough sets, Int. J. Comput. Inform. Sci. 11 (1982) 341-356.

[15] Pawlak, Z. Classification of Objects by means of Attributes, Institute for Computer Science, Polish Academy of Sciences, Report 429, 1981.

[16] Z. Wang, L. Shu, The lower and upper approximations in a group, International Journal of Mathematical and Computational Sciences, 6 (2012),158-162.

[17] Peters, J. F. Near sets, General theory about nearness of objects, Appl. Math. Sci. 1 (53), 2029-2609, 2007.

[18] Peters, J.F. Near sets, Special theory about nearness of objects, Fundam. Inform. 75 (1-4), 407-433, 2007.

[19] Peters, J. F. and Wasilewsk,P. Foundations of near sets, Information Sciences 179 , 30913109, 2009.

[20] Peters, J. F. Classification of perceptual objects by means of features, Int. J. Info. Technol. Intell. Comput. 3 (2) (2008) 1-35.

[21] Peters, J. F. Fuzzy Sets, Near Sets, and Rough Sets for Your Computational Intelligence Toolbox, Foundations of Comput. Intel. Vol. 2, SCI 202, pp. 3-25, 2009.

[22] Peters, J. F. and Ramanna, R. Feature selection: a near set approach, (in: ECML \& PKDD Workshop on Mining Complex Data, Warsaw, 2007), pp. 1-12.

[23] Peters, J. F., Naimpally, S., Applications of near sets, Notices of the Amer. Math. Soc. 59 (4), 536-542, DOI: http://dx.doi.org/10.1090/noti817, 2012.

[24] Peters, J. F. and Pal, S. K. Cantor, Fuzzy, Near, and Rough Sets in Image Analysis, (In: Pal, S.K. and Peters, J. F.(eds.) Rough Fuzzy Image Analysis: Foundations and Methodologies, CRC Pres ,Taylor and Francis Group, Boca Raton, U.S.A, 2010), 1.1-1.16.

[25] Taşbozan, H., İçen, İ., Bağırmaz, N., Özcan, A., Soft sets and soft topology on nearness approximation spaces, Filomat 31:13, 4117-4125, 2017.

[26] M.Wu, X.Xie,C.Cao, Rough subset based on congruence in a vector space, IEEE, 2008

[27] M.Wu, X.Xie, Roughness in vector spaces, IEEE, 2011

Hatay Mustafa Kemal University, Mathematics of Department, 31060, Hatay, Turkey Email address: htasbozan@mku.edu.tr 